with about equal frequency, and that these sinuses and veins are more frequently affected than any others.

From these statistics it does not appear that the lateral is more frequently affected than the other sinuses, as is generally believed. Indeed, the superior petrosal sinus was found thrombotic once more than the lateral sinus, which would lead us to infer that this disease is somewhat more inclined to spread from the middle and internal ears, than from the mastoid cells.

The frequency of involvement of the internal jugular vein, is accounted for by the easy transmission of disease from the middle and internal ear by three channels. One by the tegmen-tympani, another by a small vein passing trom the middle ear into the middle meningeal vein, and thence to the internal jugular vein, with still another means of communication by the veins before mentioned passing from the internal ear, into the superior petrosal sinus, into the lateral sinus, and into the internal jugular vein.

The venous connection between the mastoid cells, and lateral sinus, which in its turn empties into the internal jugular vein, must not be forgotten in this particular, and we therefore infer it to be but natural that the internal jugular vein should be frequently affected, as it is the dumping ground of so many venous channels.

These cases run a variable course, extending from a few hours to several weeks, but the result is usually fatal, although instances of recovery are not rare. I myself have seen several cases, of undoubted phlebitis and thrombosis, of a most severe character, where complete and permanent recoveries have occurred.

Postmortem examinations have produced frequent cases of brain thrombi, where such a condition was not suspected during life. The explanation of this phenomenon is probably the vicarious action of the rich collateral circulation. Barr reports a case where the autopsy showed complete occlusion of the lateral sinus, by fibrous bands.

Death usually takes place from metastasis, especially by embolic pleura-pneumonia, less frequently by abscess of the liver and kidney, sometimes from pyæmia. We may be sure of a fatal issue, when thrombus of the internal jugular vein is found, with metastasis of the internal organs.

We have gathered that phlebitis and thrombosis of the cerebral sinuses and veins from otorrhœa is not so fatal in its character, as cerebral abscesses, etc., from the same cause, and consequently the indications for operation, are not so imperative. We can often afford to temporize somewhat with it, treat the indications and wait for developments and active indications, before resorting to operative procedures. This is fortunate, as it is difficult to get at the focus of disease in many instances, and our methods of operation are far from being crystalized.

We may first endeavor to remove the focus of disease in the middle ear. This may be done by opening abscesses, removing granulations, polypi, or necrosed bone, and by the use of quiet, aural douches of hot water, combating any incidental conditions that may arise, and the free use of alteratives, and absorbents, such as mercury and the iodides. If these measures fail, and the symptoms continue or grow worse, the mastoid process should be freely opened, even though no signs of disease be here present.
If now, after awaiting a reasonable length of time, the symptoms show no sign of abating, we must. direct our operations directly to the sinus or vein involved. If thrombus of the lateral sinus is found, the internal jugular vein should be tied at two points in the neck, and the intervening vein tissue removed, after which the surrounding parts should be thoroughly cleansed, etc. This proceedure is necessary as an obstacle against the passage of the septic material into the general circulation.

The lateral sinus may now be exposed, by opening the skull one inch behind, and a quarter of an inch above the centre of the bony meatus. A free opening should be made, the diseased area thoroughly and antiseptically cleaned, and unhealthy tissue removed, after which it should be packed with iodoform gauze.

Ballance says that after the jugular is tied the face and lips become blue, but that it is only temporary.

Surgical interference upon the other sinuses and veins is not sufficiently crystallized to warrant extended remarks.

\section{THE ACTION OF REMEDIES.}

A paper read at the joint session of the Brainard District Medical Society und the Illinois Central District Medical Society, at Springfieid, Ill., Oct., 13-14, 1892. BY J. J. CONNER, M.D.

MEMBER OF AMERICAN MEDICAI ASSOCIATION; MEMBER OF MISSISSIPP VAIIEY MEDICAL SOCIETY ; MEMBER OF TLLINOIS CENTRAI. DDDCA SOCIETY; SECRFTARY U. S. PENSION EXAMINING BOARD, PANA, ILL.

1. The lancet; 2. Veratrum viride; 3. Opium; 4. Coal tar derivatives ; 5. Ergot ; 6. Heat (poultices) 7. Cold; 8. Quinine; 9. Ammonia; 10. Calomel.

$M r$. President and Gentlemen:-In presenting this subject to this Society it is not my purpose to traverse much of the field of materia medica, nor to notice many remedies, but to confine myself to a very few.

And in order to get a better understanding of the subject, I thought it best to choose some well known disease to base our study of remedies upon. I have therefore chosen pneumonia as a type of disease suitable for our purpose. As to whether or not pneumonia be a specific disease, having a certain definite course to run, and depending upon a particular microbe for its cause, or merely a local inflammation depending solely upon local causes, I shall not stop to discuss. I will say this, however, in the language of that Nestor of western medicine, N. S. Davis, "that the same process of reasoning that has caused many to call it (pneumonia) a general febrile disease, would equally assign all acute inflammations of important structures or organs to the same class." Neither shall I consider in detail the pathology of pneumonia, which, I doubt not, is as well understood by my hearers as by myself. I believe that we have in pneumonia-call it if you please, either a general febrile disease or a purely local manifestation-and in inflammation, precisely the same phenomena. In them both we have "a morbid process. or alteration in a part in which there is perversion of nerve action, a change in the calibre of the arterioles, capillaries and veins; an increase in quantity and a change in the quality and motion of the blood, and in the conditions and relations of the elements of the tissues; reducing them to a more embryonic state, resulting in various kinds of metamorphoses. There is a tendency to stasis of the blood, effusion of 
liquor sanguinis and leucocytes through the coats of the distended vessels; and of the red corpuscles by the rupture of their coats. There is generally a tendency to the proliferation of tissue cells and to the formation of new products, viz., lymph, pus and false membrane, and sometimes to the destruction of tissue. There is perversion of the function of the organ involved, and this process is generally attended with heat, pain, redness and swelling, and by a more or less febrile disturbance of the general system." I wish particularly to call your attention to two conditions which I believe to be the sources of danger to which a patient laboring with pneumonia is subject. I refer to the engorgement of the right side of the heart and to the distended condition of the pulmonary vessels of the lobes of the unaffected portion of the lungs. Of course, as will be noted further on, another danger accompanies or follows the great dilatation of the vessels, viz., the paralysis of the vaso-motor nerves. As is well understood, the heart, in pneumonia, has an increased amount of work thrown upon it. The affected lobes are no longer capable of allowing a circulating current of blood passing through them, but there is a stasis of the blood and consolidation of the inflammatory products which nave been thrown off during the process. Now this being so, the heart is obliged to force the same amount of blood through the unimpaired lung tissue in a certain given time as it did while all the lung substance was healthy. The unimpaired lung tissue can oxygenize only a given amount of blood in a certain time, but now all the blood must be renovated as fast and as perfectly by one lung, or by two or three lobes as it was by both lungs or all five lobes before the pneumonia began.

Now, what are the indications for treatment here? Evidently to withdraw the force of the increased circulation from the distended blood vessels in the yet unimpaired lung tissue, and to reduce the amount of work of the heart, thereby preventing distension of the capillaries and paralysis of the vaso-motor nerves, and to maintain the reserve force of the cardiac muscle, and promote resolution in the inflamed lung. If we do not relieve the pressure in the unimpaired lung tissue there will be extension of the pneumonia, because a part of the phenomena of inflammation is an increased amount of blood, in a part accompanied with rapidity of motion of the blood current. This increased blood supply and rapidity of motion in the yet unimpaired lung tissue is what leads on to inflam. mation of that portion which is still able to do all that it was accustomed to do, but if pushed to the extreme limit, will become itself involved in the morbid process and incapable of doing its usual amount of work. This extra blood supply to a Iung yet unimpaired, will, if not soon corrected, so increase the calibre of the capillaries and arterioles that the vaso-motor nerves supplying the muscular coats of these vessels will become paralyzed, rupture of the walls of these vessels will occur and the whole lung tissue will be involved in the pneumonic process.

If we had to deal with two or more reservoirs which were supplied by a large and sufficient watercourse for all proper demands upon them, and if by some mishap one or more of these reservoirs became disabled, and all the water was run into one of these reservoirs we would most certainly try to turn a part of the water in another direction, or shut off a portion of the water for fear of bursting the still unim- paired reservoir. If we for want of time or material failed in our endeavor to turn aside the rushing water, it would be necessary to tap at a certain, safe, and convenient place, the sound reservoir and relieve the undue pressure for fear the increased amount of force put upon the walls or embankments would cause them to give way. If we relieved the pressure in time we might be able to save the structure, and when the flood was over and the pressure taken off, the impaired reservoir might be repaired. So with the lungs in the storm and flood of pneumonia, we can, if the onset is not too sudden and great, cut off or relieve the pressure of the lungs by appropriate medicines and remedies, if we are given time, but if time is not given for our medicines to act, we can withdraw a portion of the blood from the turgid lungs and relieve the pressure by tapping with the lancet. The lancet is peculiarly applicable in cases of what the French are wont to call, "cerebral pneumonia," and in severe forms of pleuro-pneumonia. I have used the lancet in only a few cases, and in no case that I have used it have I regretted it; but in several cases where I did not have the courage to use it, or where such strong objections were urged against it that I deferred to them, I have been exceedingly sorry for it afterwards. In using the lancet I bleed for effect and not for quantity.

Case 1.-F. L., male, German-American, 20 years of age. While fox hunting one night sat down upon a log to rest being at the time wet with perspiration, although the ground was frozen and partly covered with snow. Twentyfour hours afterward, on the morning of March 13,1881, I was called to see him by his father. As I drove to his home about three miles in the country, I made up my mind to bleed him, as I knew he was a strong vigorous young man. I had recently been reading in the Medical and surgical Reporter, Dr. Hiram Corson's views on bleeding in pneumonia. I had pictured in my mind a flushed face, dry skin and bounding pulse as what I should find when I zot to my patient. Judge of my surprise and great disappointment when I found a pale face, moist skin, and what I now call a clogged or obstructed pulse, but which I then thought was a weak one. I was afraid to bleed him in the condition he was in, as I was a comparative stranger in that neighborhood, which had been dominated by irregulars and eclectics. He was in so grave a condition that I said to myself "if I bleed this patient and he dies it will be said that I killed him. I will therefore treat him on general principles and if he dies I shall not be blamed for his death." He steadily grew worse until the fourth morning, when he was raising a mouthful of very thin, bloody fluid almost every breath. I saw him again that night about 10 o'clock and believed he would die the next day. On the next day, being the fifth morning from my first visit, or sixth of his illness, I found him enormously improved. I saw upon entering his room, that a great change for the better had taken place, and I tell you I was frightened. I thought another doctor had been called in. I found on inquiry that at about 5 o'clock that morning the change had taken place. At that time, during a violent spell of coughing, his nose began to bleed and had bled fully a pint in a rapid stream. Nature, not being such a coward as I had been, was the doctor that had been called in, and had bled him copiously and had cured him. But I got the credit of it. This experience taught me a lesson which I shall never forget, and gave me courage.

Case 2.-Peter C., aged about 27; had been attending along with his friends and relatives, a protracted meetirg during the month of March, 1884. I was called to see him on the 16th day of the month. One of his relatives had just been buried a few days before who had died of pneumonia after an illness of about one week. This relative had lived only about forty rods from my patient's home, and my patient attended him in his late sickness. Two doctors had attended him in his illness, but they could not save him. In a consultation they had said they believed bleeding would be beneficial, but neither one dared bieed him. Peter had been sick about 36 hours when I saw him first. He was in awful distress with pleuro-pneumonia. He was sitting up in bed, being unable to lie down, cramped nearly 
double, and drawn to the right side holding it tightly with his arm. His skin was more like a frog's than a human's. It was cold, clammy and purplish. His eyes were bloodshot and staring. He was crying incessantly, "Oh! Oh! Oh!! my side! Oh! my head!" Nothing but grunt, grunt, grunt, and hollowing with his head and side. He said, "If you can give me anything, doctor, that will make me easy I want it quick, because I can't stand this pain any longer." I said, "Will you let me bleed you?" He replied,"I never was bled, but if it will ease me I will do anything." I told him that it would give instant and permanent relief. His skin was so pinched and veins so small that the blood would not run, and $I$ ordered a pan of hot water to bathe the arm in. I then bled him until the perspiration stood on his brow and he got dizzy. I laid him down and he fell into a sleep for a few minutes. He soon aroused and some one said, "Pete, what do you wish?" He said,"I have not eaten anything for a day and a night, but if you will give me a square meal I will be all right." I saw him twice after that and discharged him cured. He has told me many times since that I saved his life by bleeding him. He said that medicine would have been too slow to have saved him.

In early stages of pneumonia of moderate intensity I rely on veratrum viride as my sheet anchor. It will do good in all cases of pneumonia when seen early. In its action it is more like bleeding than any other drug that I am acquainted with. It is said to "bleed the patient into himself." That is, it dilates all the capillaries and small veins which are contracted over the entire body-especially in the skin and near the surface, and relieves the intermal pressure. It acts, I believe, directly upon the pneumogastric nerve, inhibiting or putting a break upon the heart, slowing its action, and also upon the great sympathetic nerve which governs the calibre of the blood vessels, the arterioles and capillaries especially.

In the beginning of a mild case of pneumonia I believe it possible to abort it by a judicious dose of opium or morphia. The opium acts by relieving pain thereby giving rest and quietude to the organism. Nature here steps in and finishes the job.

Some of the synthetic coal-tar derivatives will do the same thing, and partly in the same way. They act also upon the heat centre of the brain. It is admitted, I believe, that there is a "heat centre" governing the temperature of the body.

Ergot is said to act beneficially in pneumonia by contracting the vessels in the affected lungs, driving the blood out where it is not needed and distributing it to the general system. I have tried it only in a couple of cases. The theory of it acting in this way credits the drug with having a little too much dis. criminating power. By its advocates it is claimed to have what may be called intelligence enough to act only on the muscular fibre of the blood vessels of an inflamed lung, leaving untouched all the other muscular coats of the blood vessels of the system. Will it do it?

Poultices are said to act by warming the skin which relaxes the surface blood vessels, which become filled with blood, thereby drawing it off the lungs. How about the application of cold to the chest in pneumonia? How does cold relieve the pressure of the lungs? How can cold affect the vessels in the lungs when they are protected by the skin, fat, muscles, bones, fascia and pleuræ? Is it not done by depressing the heart? A better place for it then would be at the back of the head and nape of the neck in the shape of an ice bag.

Who does not give quinine in pneumonia, especially here in Illinois, but who can say why he gives it? If there is congestion of the lungs due to malarial poisoning and we have to deal with what may be called a "congestive chill," like the old practitioners used to talk so much about, all well and good, but many of us do not believe in "congestive chills" any more. Quinine acts directly upon the blood and heart. It kills the malarial parasite of the red blood corpuscle, the protozoa of Zavaran, which he discovered in 1880, and since confirmed by Osler in America and by several others. It acts upon the heart, reducing the number of beats and thereby taking the force off the lungs. It dilates the blood vessels, too, and acts also upon the heat centre of the brain. It will inhibit the heart's action in animals even after the vagi are cut. If it acts upon the heart and blood vessels as noted above, it will do good in pneumonia by draining the blood from the lungs, As quinine is a deadly weapon to the red blood corpuscle parasite, it may be that it does its good work in pneumonia by killing the diplococcus pneumoniæ of Frïnkel. To be of any utility, it must be given in large doses, at least as much as 30 , 40 or 60 grains 24 hours. I am apt to begin about midnight, as the temperature is nearly always lower at that time than any other, and give what quinine I wish my patient to have for the day in the succeeding 12.hours. I think it always does good when administered in this way. I think it beneficial to begin the treatment of pneumonia with a mercurial cathartic. The mercury does good in two ways, viz.: It unloads the prima viæ as well as any thing else, besides it prevents the formation of fibrin and helps the ammonia salts to dissolve it after it is formed. The calomel is a good diuretic, too.

Gentlemen, I thank you.

102 Second Street, Pana, 11.

MEMORANDA, PRACTICAL AND SUGGESTIVE. Read at the Annual Meeting of the New York State Medical Association,
November, 1892.

BY H. D. DIDAMA, M.D.,

OF SYRACUSE, $N$. Y.

EARLY ASPIRATION IN PLEURITIS.

In many instances pleuritis, especially the dry kind, sometimes the moist-the quantity of effusion being small-gets well with little or no treatment.

In other cases, where the attack is more severe, recovery also occurs under the use of cathartics, diuretics, sudorifics and blisters, with or without total abstinence from water or other beverage. The illness -as is well known-is apt to be prolonged; the recovery is often incomplete, and the convalescence tedious and discouraging.

Furthermore, in a large percentage of cases, the therapeutic management just indicated fails entirely to secure a favorable termination. So that the thoughtful medical attendant is sometimes perplexed to determine whether the unsatisfactory courss is due wholly to the malignancy of the disease, or in part to the imperfection of the treatment.

The consensus of modern medical opinion, as expressed in books, is that after giving the remedies a fair opportunity and a reasonably generous time to secure the absorption of the pleural effusion, if the desired object is not accomplished, or if the amount of transudation becomes rapidly so great as to imperil the life of the patient, then paracentesis should be cautiously, but to a moderate extent only, performed. The limit of this probationary period, and the danger-symptoms and signs which should be mani- 